Document downloaded from:

http://hdl.handle.net/10251/180552

This paper must be cited as:

Vraka, A.; Hornero, F.; Osca, J.; Faes, L.; Alcaraz, R.; Rieta, JJ. (2019). Assisting Electrophysiological Substrate Quantification in Atrial Fibrillation Ablation. IEEE. 1-4. https://doi.org/10.1109/EHB47216.2019.8969928

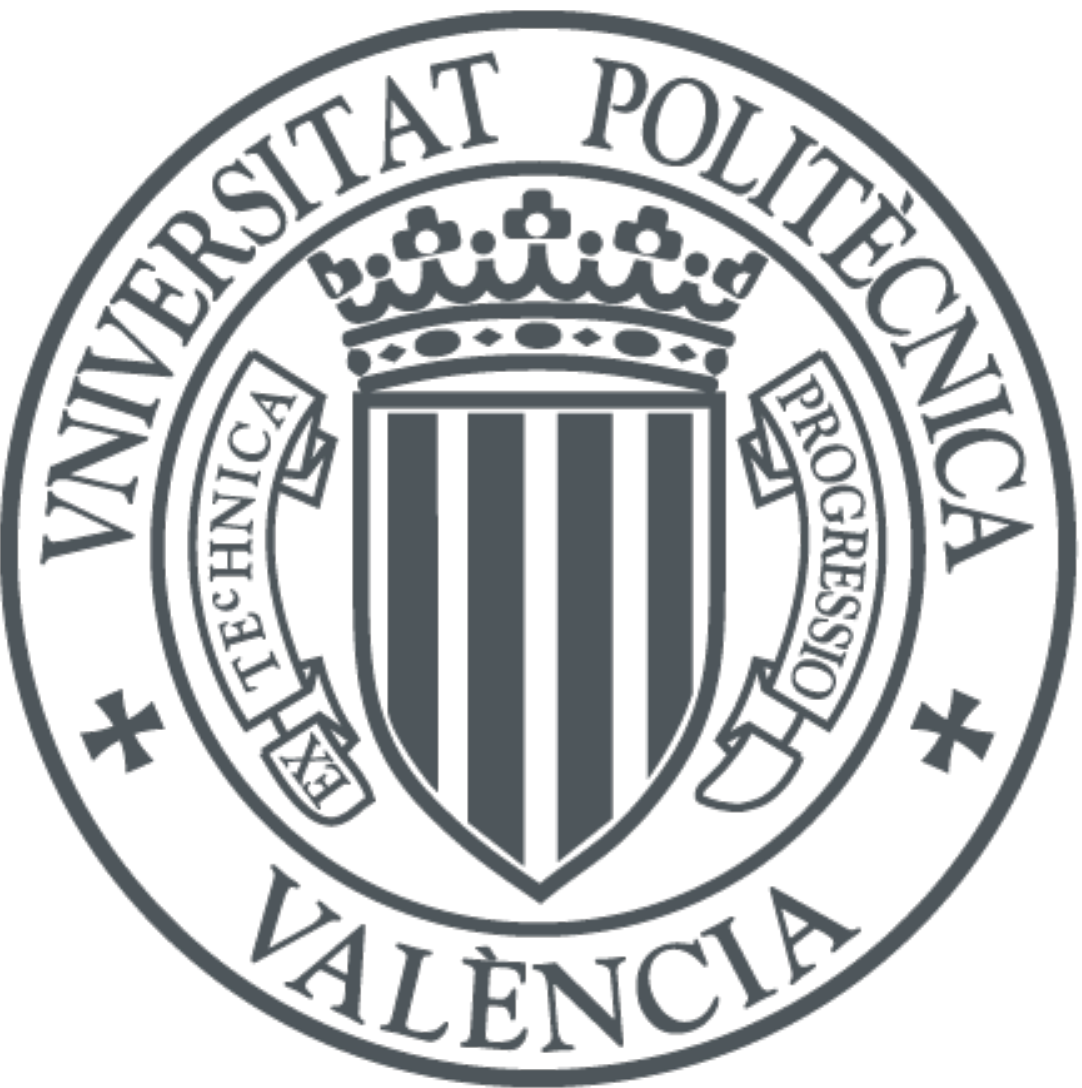

The final publication is available at

https://doi.org/10.1109/EHB47216.2019.8969928

Copyright IEEE

Additional Information 


\title{
Assisting Electrophysiological Substrate Quantification in Atrial Fibrillation Ablation
}

\author{
Aikaterini Vraka ${ }^{1}$, Fernando Hornero ${ }^{2}$, Joaquín Osca ${ }^{3}$, Luca Faes $^{4}$, Raúl Alcaraz ${ }^{5}$, José J. Rieta ${ }^{1}$ \\ ${ }^{1}$ BioMIT.org, Electronic Engineering Department, Universitat Politecnica de Valencia, Spain; \{aivra, jjrieta\}@ upv.es \\ ${ }^{2}$ Cardiac Surgery Department, Hospital Universitari I Politecnic La Fe, Valencia, Spain; hornero_fer@gva.es \\ ${ }^{3}$ Electrophysiology Section, Hospital Universitari I Politecnic La Fe, Valencia, Spain; joaquinosca@gmail.com \\ ${ }^{4}$ Department of Engineering, University of Palermo, Palermo, Italy; luca.faes@unipa.it \\ ${ }^{5}$ Research Group in Electronic, Biomedical and Telecomm. Eng., Univ. of Castilla-La Mancha, Spain; raul.alcaraz@uclm.es
}

\begin{abstract}
Catheter ablation (CA) is the most popular treatment of atrial fibrillation (AF) with good results in paroxysmal $A F$, while its efficiency is significantly reduced in persistent $A F$. With the equipment used for CA strongly depending on electrogram (EGM) fractionation quantification, the use of a reliable fractionation estimator is crucial to reduce the high recurrence rates in persistent AF. This work introduces a non-linear EGM fractionation quantification technique, which is based on coarsegrained correlation dimension (CGCD) computed over epochs of 1 second. Recordings were firstly normalized, denoised and lowpass filtered. The final CGCD value was calculated by the median CGCD value of all the epochs that a recording consisted of. Results were evaluated on three groups. Groups 1 and 2 contained 24 high-quality and 119 mid-range EGMs, respectively, manually pre-classified by AF types following Wells' criteria, then classified according to their CGCD values. 20 pseudo-real Type IV EGMs formed group 3 that was also automatically classified by AF type. In Groups 1 and 2, classification accuracy was $100 \%$ and $84-85.7 \%$, respectively, using 10 -fold cross-validation. The receiver-operating characteristics (ROC) analysis for highly fractionated EGMs, showed $100 \%$ specificity and sensitivity in Group 1 and $87.5 \%$ specificity and 93.6\% sensitivity in Group 2 . CGCD was always consistent with the fractionation degree of EGMs. $100 \%$ of the EGMs in Group 3 were correctly identified as Type IV AF. High accuracy results indicate that the method can estimate precisely the AF Type and detect the existence of AF Type IV cases. Both things are crucial in assisting improved substrate mapping during $\mathrm{CA}$ procedures of persistent $\mathrm{AF}$.
\end{abstract}

Keywords-Atrial fibrillation; catheter ablation; electrogram fractionation; correlation dimension.

\section{INTRODUCTION}

Atrial fibrillation (AF) is the most common cardiac arrhythmia in the developed countries, associated with high mortality risk [1]. Challenges regarding AF deal with understanding the AF perpetuating mechanisms and developing the appropriate therapeutic scheme, adapting to each patient's needs.

Common AF treatments include electrical and pharmacological cardioversion and surgical or catheter ablation (CA) [1]. Due to the significance of pulmonary veins (PVs) in the generation of fibrillatory activity [2], CA of PVs, called pulmonary vein isolation (PVI), is considered the first-line AF therapy [3]. High-efficiency of PVI is limited to paroxysmal $\mathrm{AF}$ patients whereas recurrence rates of PVI in persistent AF (peAF) cases remain high, being the structural remodelling of peAF the most probable reason [4].

Fibrosis is considered the principal remodelling phenomenon that favors the perpetuation of AF [3], [4]. Works orientated to detecting fibrotic tissue have recently been focused on the detection and study of dynamic phenomena, such as low voltage (LV) areas and atrial regions with high electrogram fractionation [3].

A vast amount of studies have dealt with the detection and ablation of areas presenting EGMs with high fractionation, commonly known as complex fractionated atrial electrograms (CFAEs). While CFAEs have often been a cause of argument either regarding its own definition [5], [6] or the efficiency of the CFAEs ablation [7]-[9], the need for a precise and reliable definition as well as estimation of such types of EGMs is emerging. To this respect, correlation dimension and its alternative version used in biomedical signals, coarse-grained correlation dimension (CGCD), are two very interesting chaosbased approaches [10] that can be interesting tools in assessing the electrophysiological substrate of atrial fibrillation. In fact, both have been previously used in order to assess the organization of AF dynamics [11], [12].

This work presents a customized version of CGCD, designed to operate in very short time and to adapt to any signal length. This method was proved to efficiently express the fragmentation level of bipolar EGMs and correctly classify patients by AF types, according to Wells' criteria [13]. Rapid changes in AF type were easily detected and AF Type IV was successfully diagnosed, while in traditional analysis would be misinterpreted as AF Type II or III . High performance in short-time and adaptability suggest this fractionation estimator to be implemented on current medical devices. 


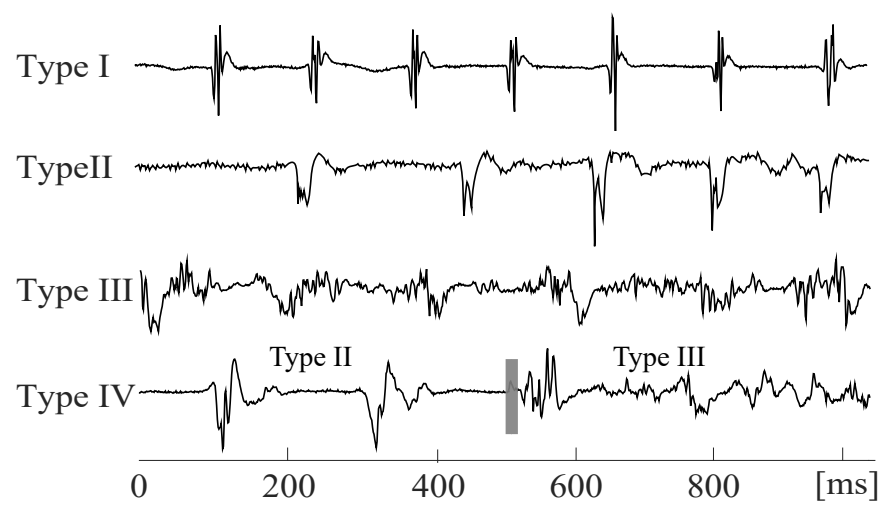

Fig. 1. Example of bipolar AF EGMs of different types. AF Type IV consists of alternating Type I/II and Type III segments.

\section{MATERIALS}

The database employed in this study consisted of 119 10-s duration bipolar EGMs of 22 peAF patients undergoing CA for the first time. Data was obtained using a CardioLab ${ }^{\circledR}$ system (General Electric, Wauwatosa, WI, USA) with a sampling frequency of $1 \mathrm{kHz}$. EGMs were visually inspected and classified according to their AF Type following Wells' criteria [13]. Figure 1 shows an example of different AF types of the EGMs processed. In total, 11 EGMs were classified as Type I, 36 as Type II and 72 as Type III. Visual classification by AF type can be confusing in cases that an EGM does not clearly belong to an AF type. In order to make a fair assessment of CGCD as a fractionation index, 8 EGMs from each category were selected by expert phisicians as the most representative of their type. Additionally, 20 pseudo-real AF Type IV EGMs were created by the concatenation of parts of the real EGMs.

Data analysis has been performed on three groups. Group 1 consisted of the 24 most representative EGMs, eight of each type, as explained previously. Group 2 consisted of all the electrograms in the database, while Group 3 consisted of the artificial Type IV electrograms.

\section{METHODS}

\section{A. Data preprocessing and analysis}

All preprocessing and evaluation steps were performed using MATLAB 2018a software (The Mathworks ${ }^{\circledR}$ ). Signal normalization by its root mean square (RMS) value was the first step to be performed, in order to minimize the influence of the signal amplitude on CGCD. RMS value of each signal depends on its properties and the choice of this normalization parameter was made in order to keep the information of each time-series intact.

After that, signal preprocessing continued by using a 3rd order Butterworth lowpass filter with cut-off frequency at $300 \mathrm{~Hz}$ and a wavelet-based denoising technique which reduced effectively high frequency noise [14]. Finally, EGMs were segmented to one second intervals, so that any changes in the pattern of AF can be captured rapidly and the CGCD computational parameters can be used regardless of the signal length.

For the computation of CGCD, a $N$-points long signal $X=\left(x_{1}, x_{2}, \ldots, x_{N}\right)$ needs to be firstly reconstructed in the $m$ dimensional phase-space [15] using a time delay $\tau$ between vectors [11], therefore

$$
\mathbf{Y}_{p}^{(m)}=\left(x_{p}, x_{p+\tau}, x_{p+2 \tau}, \ldots, x_{p+(m-1) \tau}\right),
$$

where $m=1,2,3 \ldots$ and $p=1,2, \ldots, N-(m-1) \tau$.

Then, the correlation integral $C^{(m)}(r)$ is calculated, as the proportion of pairs of vectors that are closer to each other than a distance $r$, thus

$$
\begin{aligned}
C^{(m)}(r)= & \frac{2}{N_{\text {ref }}\left(N_{\text {ref }}-1\right)} \sum_{i=1}^{N_{\text {ref }}} . \\
& \cdot \sum_{j>i}^{N_{\text {ref }}} \Theta\left(r-\left\|Y_{i}^{(m)}-Y_{j}^{(m)}\right\|\right),
\end{aligned}
$$

where $\Theta$ is the Heaviside function, $\|\cdot\|$ is the Euclidean distance of each pair chosen and $N_{r e f}$ is the number of reference points, as a chosen number of the $N-(m-1)$ vectors of Equation 1.

Correlation dimension is calculated from the saturation areas on the double logarithmic plot of $C^{(m)}(r)$ as a function of $r$, plotted in sequential embedded dimensions from $m=1,2, \ldots, 20$ [12]. In biosignals, CGCD, a rough estimator of correlation dimension which does not express precisely the dimension of the dynamics but serves as a comparative measure between dynamics of different levels of organization [11], [12], can be used by setting a fixed embedded dimension $m$ and a finite resolution distance $r_{c g}$

$$
C G C D^{(m)}\left(r_{c g}\right)=\frac{\ln \left[C^{(m)}\left(r_{c g}\right)\right]}{\ln \left(r_{c g}\right)} .
$$

CGCD was computed for each time-series at the segmented one second intervals and then the final CGCD value was obtained by the median index of all the intervals. Parameters selection of CGCD were made so that the comparative analysis of fractionation of each EGM is optimal. Firstly, the time when mutual information dropped to its first minimum was calculated. Analysis indicated that for our dataset, this value was for $\tau=8 \mathrm{~ms}$. $N_{\text {ref }}$ was firstly set equal to $1 / 3$ of signal length [16], that is, in our case, 334 points. Distance $r_{c g}$ was computed for each signal equal to half of its standard deviation, normalized by its peak-to-peak amplitude [12]. Finally, CGCD was computed for different embedded dimensions from $m=1,2, \ldots 20$ and the dimension providing the most discriminative power and, at the same time, avoiding infinite CGCD values was selected. In this case, dimension $m=4$ was the optimal choice.

After setting the parameters, different sizes of reference points $N_{\text {ref }}$ were tried in order to find the optimal value. $N_{\text {ref }}$ was firstly increased to $1 / 2$ of the signal length and then the maximum number of $N_{r e f}$ was used for the analysis. However, discrimination through fractionation levels was not improved, 
TABLE I

RESULTS OF ROC ANALYSIS FOR GROUPS 1 AND 2 IN THE DATABASE. AUC STANDS FOR AREA UNDER THE ROC CURVE.

\begin{tabular}{cccc}
\hline AF Type & AUC & Sensitivity & Specificity \\
\hline \multicolumn{4}{c}{ Group 1 } \\
Type I vs II/III & 1.0000 & $100 \%$ & $100 \%$ \\
Type III vs I/II & 1.0000 & $100 \%$ & $100 \%$ \\
\hline \multicolumn{4}{c}{ Group 2 } \\
Type I vs II/III & 0.9907 & $97.22 \%$ & $100.00 \%$ \\
Type III vs I/II & 0.9515 & $87.50 \%$ & $93.62 \%$ \\
\hline
\end{tabular}

TABLE II

CLASSIFICATION ACCURACY BY COARSE DECISION TREE FOR GROUPS 1 AND 2 TOGETHER WITH THE NUMBER OF EGMS WRONGLY CLASSIFIED.

\begin{tabular}{cccc}
\hline Group & Nr of EGMs & Accuracy & Wrongly classified \\
\hline 1 & 24 & $100 \%$ & 0 \\
2 & 119 & $84-85.7 \%$ & 17 \\
\hline
\end{tabular}

while execution time was significantly increased. The number of reference points was therefore kept to $N_{\text {ref }}=334$.

\section{B. Evaluation}

Groups 1 and 2 were classified by AF types according to their CGCD values by a one-vs-all receiver-operating characteristics (ROC) curve, firstly used in order to define the upper threshold CGCD value for AF Type I. In the same way, the lower threshold CGCD value for AF Type III was calculated. ROC characteristics were then used in order to see how clearly AF Type I and III can be discriminated from the rest of the AF types. Discriminative power of CGCD was also assessed by a coarse decision tree with a maximum of 2 number of splits and 10-fold cross validation,making use of the Classification Learner App (MATLAB 2018a \& Simulink 9.1).

The ability of the method to detect AF Type IV cases was investigated by an algorithm that was developed especially for that purpose. CGCD analysis was firstly performed as described in section III-A. Then, the AF Type of signals was calculated at each epoch, using the thresholds obtained by the decision tree analysis on Group 2. The final AF Type of an EGM was defined by the median CGCD value of all its epochs unless a signal was found to consist of segments of both AF Type III and AF Type I or II. In such case, the EGM was finally assigned to AF Type IV. Figure 2 shows the steps followed for the Group 3 analysis. Performance classification on this Group was expressed as the percentage of the EGMs correctly classified as AF Type IV.

\section{RESUlts}

Receiver operating characteristic (ROC) analysis performed on Groups 1 and 2 can be observed in table I. Mean and standard deviations of all three groups are shown on the boxplots of figure 3. As can be seen, EGMs of Group 1 can be completely distinguished by AF types using CGCD. In Group 2, ROC analysis showed very satisfactory results. Although there are some overlapping CGCD values between

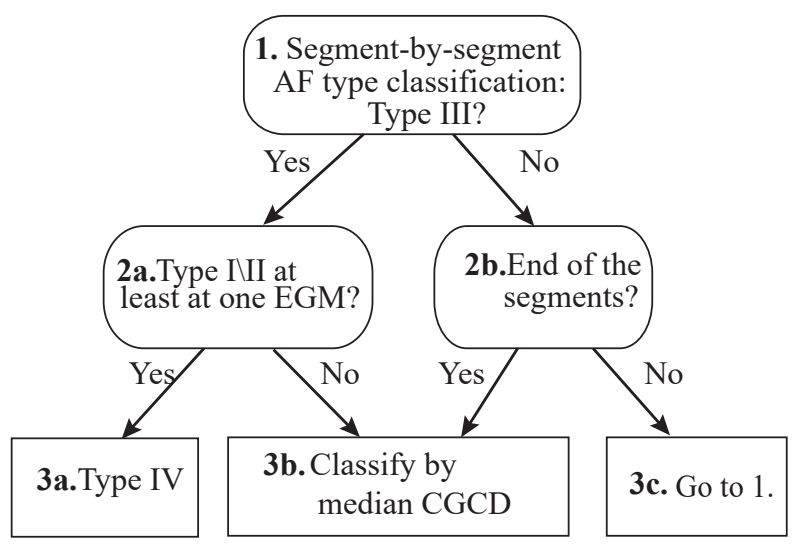

Fig. 2. Illustration of the algorithmic steps for the detection of AF Type IV on Group 3.

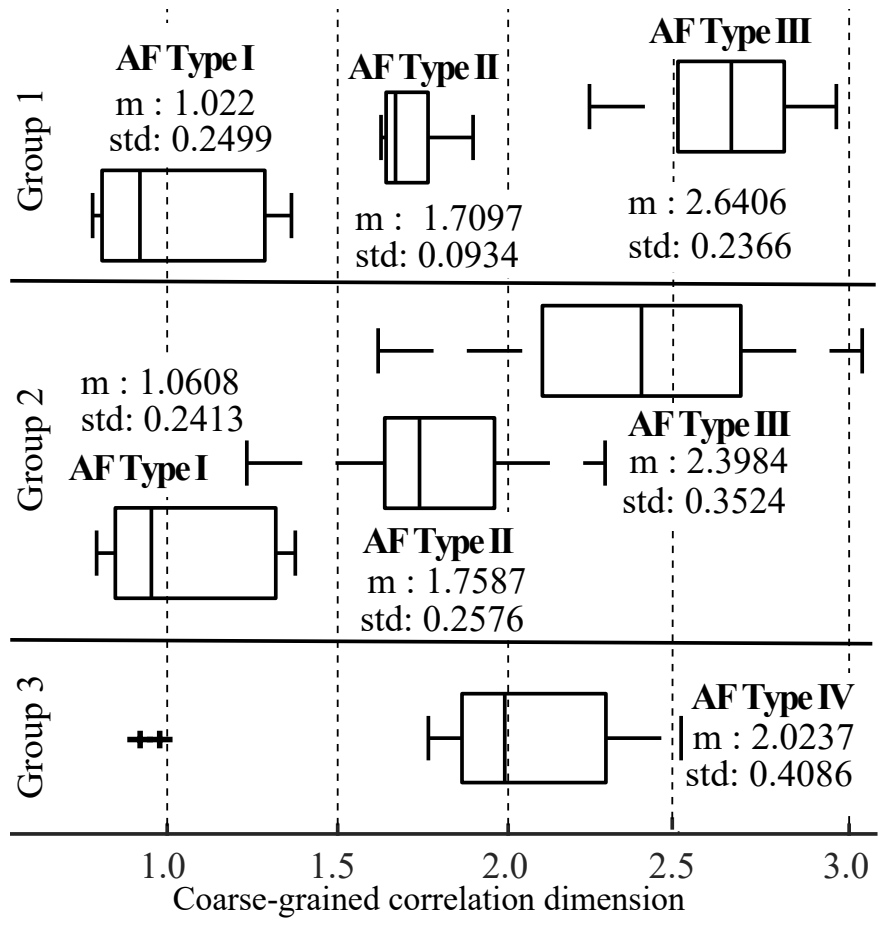

Fig. 3. Boxplots illustrating the distribution of the values of AF types at each one of the Groups. m:mean; std: standard deviation.

the three AF types, AF Type I can be perfectly distinguished with $97.22 \%$ Sensitivity and $100 \%$ Specificity, while AF Type III can be discriminated by the other two AF types with $87.5 \%$ Sensitivity and $93.62 \%$ Specificity.

As can be seen from figure 3, CGCD values of AF Type IV are located between CGCD values of AF types II and III. This means that in a compact analysis, without the use of one second segments, EGMs of AF Type IV would be misinterpreted either as AF Type II or III. Table II shows the classification accuracy of Groups 1 and 2. For Group 3, all EGMs were correctly classified as AF Type IV. 


\section{DISCUSSION}

The work presented combines previously studied concepts with new preprocessing and analysis techniques, with the aim to improve the mapping process of the AF substrate. To the best of our knowledge, this is the first study to employ CGCD for the estimation of the fractionation level of bipolar EGMs and to analyze segments of one second length, confronting some common problems regarding the EGM fractionation analysis. Stability of the recording catheter appears to be such an issue [17] that can be solved by using short-time intervals.

Detection of AF Type IV is a rather complicated task. Analysis performed on this study showed that CGCD values of AF Type IV lie dispersedly between AF types II and III. Consequently, a strategy without signal segmentation would wrongly classify the corresponding EGMs as either of the two above-mentioned AF types. Analysis in short-length epochs on the contrary, allowed the quick capture of changes in signal's morphology and as a result, the rapid detection of AF Type IV.

Choice of the signal reconstruction parameters should be done with caution and depend on the data size [18]. While longer segments are preferred, attractor dynamics can be preserved in short signals. Since different studies analyze timeseries of different size, the use of the same parameters as a common reference would serve both for comparative reasons and for the optimization of the results. Segments of one second duration can effectively adapt to the needs of each study, while the parameters with the highest discriminative power could be incorporated into the medical devices. As signal amplitude may affect CGCD values, RMS normalization allows an amplitude-independent comparison, leading to more robust results.

\section{CONCLUSIONS}

The proposed customized method demonstrated competent results. AF fractionation levels were in all cases in consistency with the CGCD values. AF Type IV was correctly detected in all the cases. As this method appears to be quick, robust and independent of common procedural issues such as catheter stability, it could contribute to AF mapping and assist CA procedures.

\section{ACKNOWLEDGMENTS}

Research supported by grants DPI2017-83952-C3 from MINECO/AEI/FEDER UE, SBPLY/17/180501/000411 from JCCLM and AICO/2019/036 from GVA.

\section{REFERENCES}

[1] P. Kirchhof, S. Benussi, D. Kotecha, and et al., "2016 ESC Guidelines for the management of atrial fibrillation developed in collaboration with EACTS. The Task Force for the management of atrial fibrillation of the European Society of Cardiology (ESC). Developed with the special contribution of the European Heart Rhythm Association (EHRA) of the ESC ," Europace, vol. 18, no. 11, pp. 1609-1678, Nov. 2016.

[2] M. Haïssaguerre, P. Jaïs, D. C. Shah, A. Takahashi, M. Hocini, G. Quiniou, S. Garrigue, and A. Le Mouroux, "Spontaneous Initiation of Atrial Fibrillation by Ectopic Beats Originating in the Pulmonary Veins," The New England Journal of Medicine, vol. 339, no. 10, pp. 659-666, 1998
[3] A. Verma and L. Macle, "Persistent atrial fibrillation ablation: Where do we go from here?" Canadian Journal of Cardiology, vol. 34, no. 11, pp. $1471-1481,2018$.

[4] J. Kornej, K. Schumacher, S. Zeynalova, P. Sommer, A. Arya, M. Weiß, C. Piorkowski, and D. Husser, "Time-dependent prediction of arrhythmia recurrences during long-term follow-up in patients undergoing catheter ablation of atrial fibrillation: The leipzig heart center af ablation registry," Scientific Reports (Nature Publisher Group), vol. 9, pp. 1-7, 05 2019.

[5] G. Lee, K. Roberts-Thomson, A. Madry, S. Spence, A. Teh, P. M. Heck, S. Kumar, and P. M. Kistler, "Relationship among complex signals, short cycle length activity, and dominant frequency in patients with long-lasting persistent AF: A high-density epicardial mapping study in humans," Heart Rhythm, vol. 8, no. 11, pp. 1714-1719, 2011.

[6] T. P. Almeida, G. S. Chu, J. L. Salinet, F. J. Vanheusden, X. Li, J. H. Tuan, P. J. Stafford, G. A. Ng, and F. S. Schlindwein, "Minimizing discordances in automated classification of fractionated electrograms in human persistent atrial fibrillation," Medical and Biological Engineering and Computing, vol. 54, no. 11, pp. 1695-1706, 2016.

[7] B. Sunsaneewitayakul, M. Schwab, C. Khunnawat, T. Vasavakul, J. McKenzie, T. Ngarmukos, E. Kosar, and K. Nademanee, "A new approach for catheter ablation of atrial fibrillation: mapping of the electrophysiologic substrate," Journal of the American College of Cardiology, vol. 43, no. 11, pp. 2044-2053, 2004.

[8] Y. J. Lin, M. T. Lo, S. L. Chang, L. W. Lo, Y. F. Hu, T. F. Chao, F. P. Chung, and J. N. Liao, "Benefits of Atrial Substrate Modification Guided by Electrogram Similarity and Phase Mapping Techniques to Eliminate Rotors and Focal Sources Versus Conventional Defragmentation in Persistent Atrial Fibrillation," JACC: Clinical Electrophysiology, vol. 2, no. 6, pp. 667-678, 2016.

[9] S. Ammar-Busch, T. Reents, S. Knecht, T. Rostock, T. Arentz, M. Duytschaever, T. Neumann, and B. Cauchemez, "Correlation between atrial fibrillation driver locations and complex fractionated atrial electrograms in patients with persistent atrial fibrillation," PACE - Pacing and Clinical Electrophysiology, vol. 41, no. 10, pp. 1279-1285, 2018.

[10] J. E. Skinner, M. Molnar, T. Vybiral, and M. Mitra, "Application of chaos theory to biology and medicine," Integr Physiol Behav Sci, vol. 27, no. 1, pp. 39-53, 1992.

[11] B. P. Hoekstra, C. G. Diks, M. A. Allessie, and J. De Goedb, "Nonlinear Analysis of Epicardial Atrial Electrograms of Electrically Induced Atrial Fibrillation in Man," Journal of Cardiovascular Electrophysiology, vol. 6, no. 6, pp. 419-440, 1995.

[12] A. Luca, A. Buttu, E. Pruvot, P. Pascale, L. Bisch, and J. M. Vesin, "Nonlinear analysis of right atrial electrograms predicts termination of persistent atrial fibrillation within the left atrium by catheter ablation," Physiological Measurement, vol. 37, no. 2, pp. 347-359, 2016.

[13] J. L. Wells, R. B. Karp, N. T. Kouchoukos, W. A. Maclean, T. N. James, and A. L. Waldo, "Characterization of Atrial Fibrillation in Man: Studies Following Open Heart Surgery," Pacing and Clinical Electrophysiology, vol. 60, no. 3, pp. 426-438, 1978.

[14] M. Martínez-Iniesta, J. Ródenas, R. Alcaraz, and J. J. Rieta, "Waveform Integrity in Atrial Fibrillation: The Forgotten Issue of Cardiac Electrophysiology," Annals of Biomedical Engineering, vol. 45, no. 8, pp. 1890-1907, 2017.

[15] F. Takens, "Detecting strange attractors in turbulence," Dynamical sys tems and Turbulence, vol. 898, pp. 366-381, 1981.

[16] J. Theiler, "Statistical precision of dimension estimators," Physical Review A, vol. 41, no. 6, pp. 3038-3051, 1990.

[17] V. Křemen, L. Lhotská, M. MacAš, R. Čihák, V. Vančura, J. Kautzner, and D. Wichterle, "A new approach to automated assessment of fractionation of endocardial electrograms during atrial fibrillation," Physiological Measurement, vol. 29, no. 12, pp. 1371-1381, 2008.

[18] R. G. Huffaker, "Phase Space Reconstruction from Time Series Data: Where History Meets Theory," International European Forum on System Dynamics and Innovation in Food Networks, 2010 International European Forum, February 8-12, 2010, Innsbruck-Igls, Austria 100455, 2010. [Online]. Available: https://ideas.repec.org/p/ags/iefi10/100455. html 